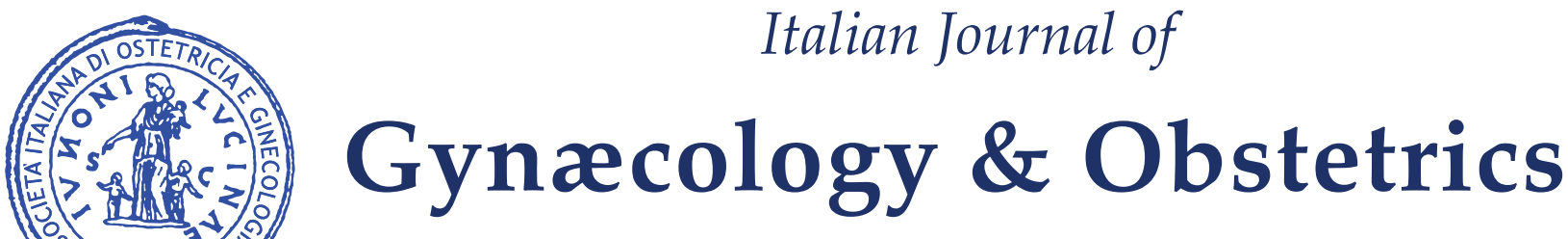

March 2021 - Vol. 33 - N. 1 - Quarterly - ISSN 2385 - 0868

\title{
Supra- versus Infra-panniclular skin incision in cesarean section: surgical techniques
}

\author{
A. S. El-Agwany \\ Department of Obstetrics and Gynecology, Faculty of Medicine, Alexandria University, Alexandria, Egypt
}

Corresponding Author: Ahmed Sami El-Agwany

E-mail: ahmed.elagwany@alexmed.edu.eg
Key words:

Cesarean section; surgery; panniculus; skin incision; delivery.

Copyright 2021

DOI: $10.36129 /$ jog.33.01.08

To the Editor,

Cesarean section is rising now with an estimated prevalence in the UK about $25 \%$ of deliveries and in Egypt, a developing country, it is around 55\% and maybe more. Obesity and overweight patients with BMI over 30 are rising now due to poor lifestyle and unhealthy diet. Obesity prevalence in the UK is around $30 \%$ and it is rising. So, we commonly encounter obese patients scheduled for cesarean section whether as elective or emergency cases. Cesarean section is high in obese women due to poor progress of labor, large baby, abnormal position and presentation.

Regarding the cesarean section, the available evidence and the number of questions still debated about the best surgical approach, there is no standard practice for cesarean section technique. As the following examples shown here, there is no evidence or effect for peritoneal closure to justify the additional time and use of suture material along with no effect on fever or healing. Also, no difference between a sharp or blunt extension of the uterine incision apart from the associated lower blood loss and lower need for blood transfusion in the blunt type. Catgut closure versus polygalactin was associated with a lower blood transfusion and complications in the catgut group. Single-layer closure can lower blood loss with respect to a double-layer one. Pelvic pain, adhesions, infertility, deep dyspareunia, hysterectomy, and outcomes of subsequent pregnancies following blunt versus sharp entry, exteriorization of the uterus versus intra-abdominal repair, single versus double-layer closure of the uterus, closure versus non-closure of the peritoneum and chromic catgut versus polygalactin were evaluated and there was no difference. The type of the technique used to perform the cesarean section does not influence significantly the outcome. Double-layer closure is associated with a thicker 35-38 weeks third-trimester lower uterine segment thickness $>2.0 \mathrm{~mm}$ but no difference in the uterine dehiscence or uterine rupture. The debate is still open regarding residual myometrial thickness alone could be the main important risk factor or not. Monofilament sutures may improve uterine scar healing without increasing costs, operation time by a larger thickness of the multifilament suture (1).

Obstetricians performing cesarean section should evaluate for risk factors for complications as bladder 
injury, bowel injury, bleeding, etc., and promptly recognize and repair the lesion. Identification and immediate repair are of paramount importance reducing the risk of further surgical procedures and complications. Previous cesarean obesity and peritoneal adhesions are the main risk factors for complications (2). Regarding obesity, obese patients have high body fat in the abdomen around the viscera and in the pelvis, so obstruction during delivery or soft tissue dystocia is common in opposition to the old beliefs that fat tissue weight on the pelvis can widen the pelvic dimension by pressure and favors easy delivery. Excess fatty tissue can hinder delivery by soft tissue obstruction.

Cesarean section in obese women is technically difficult due to deep pelvis, deep suturing needed, long instruments needed, an engorged uterus encountered, varicose veins especially in the broad ligament with high risk for injury and hematoma formation, even on gentle manipulation without traumatic injury and infection is also common.

A skin infection can occur in 10\% after the cesarean section and is more in obese women. The subpannicular fold is so wet from sweat and so is rich in bacteria and also poorly related hygiene increases susceptibility to infection.

Regarding surgical technique in obese women, the skin incision can be supra or infra-pannicular one, we are going to discuss them in detail and their role along with the techniques to avoid skin infection in these women.

The supra-pannicular skin incision is usually performed above the panniculus through the large subcutaneous fat as a high transverse or vertical incision below the umbilicus after pulling the panniculus down. The limit of the vertical incision is the felt symphysis pubis through the panniculus and the transverse incision is wider due to difficult fetal extraction and delivery from the deep abdominal and uterine cavity.

The infra-pannicular skin incision is the transverse one below the panniculus away from the reflection of the panniculus at the usual Pfannenstiel incision near symphysis pubis but away from the sensitive pubic hair area. The fatty layer here is thinner and a smaller narrower incision is used, and the pelvic cavity is reached more easily and quickly.

We may need to elevate the panniclus by an adhesive non-allergic tape to the top or sides of the operating table. This limits assistant surgeon fatigue while raising the panniclus during the potentially prolonged surgery-suprapannus transverse skin incision is associated with more bleeding on subcutaneous dissection, infection, more tissue dissection, need for drain seroma formation, subtheath hematoma and dehiscence related to infection and poor cosmetically. Regarding the vertical one it is associated with more pain, difficult breathing, difficult early ampulation and not good cosmetically. It can be used with abdominal masses that difficult to be approached from the pelvis (figures 1, 2).

The infra-pannus skin incision is associated with a lower incidence of skin infection due to the thin fatty layer, rapid entry, less vascular area, away of the moist reflection, interrupted skin suture, less bleeding and hematoma formation and shorter duration of surgery. Daily cleaning after exposure after $24 \mathrm{hrs}$ and keeping the area dry from sweat and water especially with perineal hygiene is very important. Antibiotic prophylaxis is needed for 7 days especially in diabetics with poor control.

If the panniculus is redundant down after delivery covering the incision site, we proceed with interrupted sutures. If after delivery the panniclus is above the incision site and will not go down on motion then subcuticular sutures can be used and management is as non-obese patients.

Regarding techniques for obese patients' surgical technique, we incise the subcutaneous tissue sharply at the midline then bluntly with fingers laterally exposing the rectus sheath. Avoid dissecting the subcutaneous layer below the skin edges to

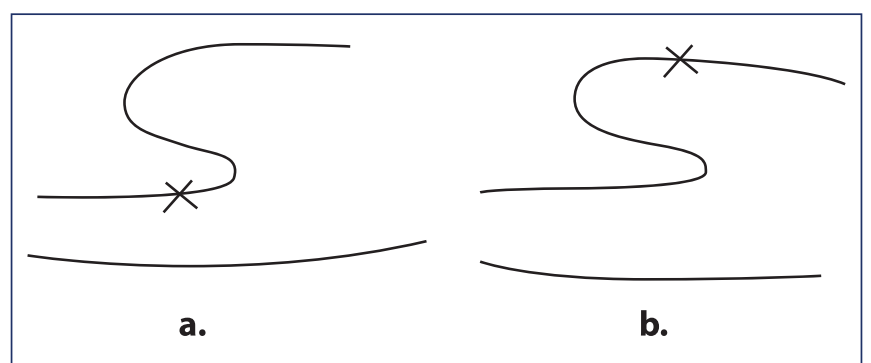

Figure 1. Suprapannicular versus infrapannicular skin incision.

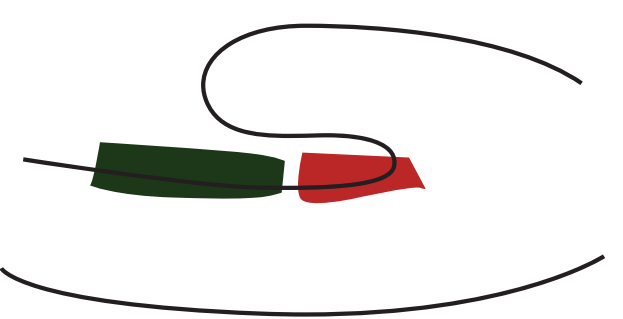

Figure 2. Avoid the red area and incise in green area that can be uncovered by a pulling up dressing or at the exposed area below. 
avoid seroma formation. Incise the sheath sharply at midline then bluntly till the skin edges at the angles and not beyond to avoid puckering of skin on suturing the sheath or missing the beginning of sheath on suturing leaving a defect. Make the skin incision wide enough to avoid dissecting the rectus sheath from muscle and provide more space for the fetal delivery. This will prevent sub-rectal sheath hematoma that can present early as hematoma or hypovolemic shock as hematoma can extend down to the vulva that can be seen easily there as thinner skin than the thicker abdominal one that can accommodate huge amount before being visualized or can appear commonly as skin discoloration and infection after two weeks-incise the uterus high up at the level of the skin incision not low down as difficult suturing later as difficult deep suturing in the deep pelvis by large skin thickness. It is better to take care of the engorged large varicose veins of the broad ligament. If injured, ligate the ligament down below them to avoid extension of hematoma retroperitoneal leaving the ovary with blood supply from the uterus even if it shows black spots of venous congestion, it can regain vitality once collaterals and uterine supply increase over time as long as no ovarian ligament ligated. Also, this can be managed by ligating part of the broad ligament, if not largely affected and the ovarian vessels can be preserved. It is better to proceed with uterine exteriorization to suture the uterus easily instead of doing that in the deep pelvis with short instruments. The uterus is commonly becoming congested or engorged on exteriorization for a long period, so reduction may be difficult or traumatic and it is better to be done gently with a towel and a retractor along with ensuring sub sheath hemostasis. No drains should be left in the subcutaneous tissue to avoid infection but hemostasis is important and closure of the dead space, especially below the skin angles. DVT prophylaxis is essential. Long-standing diabetes is commonly associated with vasculitis and fibrosis of the tissues so tissues are usually tough especially the subcutaneous tissue on incision and can tear easily as non-elastic and dissect more difficulty with a scalpel as fibrosis $(3,4)$.

We need to incorporate these technical tips in the learning of the residents and physicians. The European training programs in ob-gyn still vary significantly. A new European training curriculum encompassing all the competencies required of a newly trained specialist is an essential step for harmonization of training and standardization of care (5).

The professional competence scale consisted of eight dimensions with a total of 51 items was proposed that can be used for training and evaluation of physicians these dimensions are essential medical knowledge public health or social science essential clinical skills advanced clinical skills communication skills advanced study skills critical thinking and adaptation and professionalism (6).

\section{ETHICS}

All procedures performed in studies involving human participants were in accordance with the ethical standards of the institutional and/or national research committee and with the 1964 Helsinki declaration and its later amendments or comparable ethical standards. Informed consent was obtained from the patient included in the study.

\section{CONFLICT OF INTERESTS}

The author declares that he has no conflict of interests. 


\section{REFERENCES}

1. Laganà AS, Cromi A, Tozzi R, Franchi M, Lukanović D, Ghezzi F. Uterine Scar Healing After Cesarean Section: Managing an Old Surgery in an Evidence-Based Environment. J Invest Surg 2019;32(8):770-2.

2. Franchi M, Raffaelli R, Baggio S, et al. Unintentional transvesical caesarean section: incidence, risk factors, surgical technique and post-operative management. Eur J Obstet Gynecol Reprod Biol 2019;236:26-31.

3. Machado LS. Cesarean section in morbidly obese parturients: practical implications and complications. N Am J Med Sci 2012;4(1):13-8.
4. Bell J, Bell S, Vahratian A, Awonuga AO. Abdominal surgical incisions and perioperative morbidity among morbidly obese women undergoing cesarean delivery. Eur J Obstet Gynecol Reprod Biol 2011;154:16-9.

5. Aabakke AJ, Kristufkova A, Bune LT, et al. European trainees support the new European curriculum in Obstetrics and Gynaecology. Eur J Obstet Gynecol Reprod Biol 2016;203:335-6.

6. Cheng X, Chen J. An Exploration of Medical Education in Central and Southern China: Measuring the Professional Competence of Clinical Undergraduates. Int J Environ Res Public Health 2019;16(21):4119. 